\title{
Crystallization and structure analysis of the core motif of the Pks13 acyltransferase domain from Mycobacterium tuberculosis
}

\author{
Mingjing Yu ${ }^{1}{ }^{\text {, Chao Dou }}{ }^{1}$, Yijun Gu ${ }^{2}$, Wei Cheng ${ }^{\text {Corresp. }}{ }^{1}$ \\ ${ }^{1}$ Department of Respiratory and Critical Care Medicine, West China Hospital, Sichuan University, Chengdu, Sichuan, People's Republic of China \\ 2 National Center for Protein Science, Shanghai, People's Republic of China \\ Corresponding Author: Wei Cheng \\ Email address: chwlab@163.com
}

Type I polyketide synthase 13 (Pks13) is involved in the final step of the biosynthesis of mycolic acid in Mycobacterium tuberculosis (Mtb). Recent articles have reported that Pks13 is an essential enzyme in the mycolic acid biosynthesis pathway, and it has been deeply studied as a drug target in TB. We report a high-resolution structure of the acyltransferase (AT) domain of Pks13 at $2.59 \AA$ resolution. Structural comparison with the full-length AT domain (PDB code, 3TZW and 3TZZ) reveals a different orientation of the Cterminal helix and rearrangement of some conserved residues. 


\section{Crystallization and structure analysis of the core motif of the}

\section{Pks13 acyltransferase domain from Mycobacterium}

\section{3 tuberculosis}

4 Mingjing $\mathrm{Yu}^{\mathrm{a}}$, Chao Dou ${ }^{\mathrm{a}}$, Yijun $\mathrm{Gu}^{\mathrm{b}}$ and Wei Chenga*

5 a Department of Respiratory and Critical Care Medicine, West China Hospital, Sichuan University, 37 Guoxue

6 Road, Chengdu, Sichuan, 610041, People's Republic of China

7 b National Center for Protein Science Shanghai China, People's Republic of China

$8 *$ *Correspondence email: $\underline{\text { cwlab@163.com }}$

\section{Abstract}

11 Type I polyketide synthase 13 (Pks13) is involved in the final step of the biosynthesis of mycolic acid in Mycobacterium tuberculosis (Mtb). Recent articles have reported that Pks13 is an essential enzyme in the mycolic acid biosynthesis pathway, and it has been deeply studied as a drug target in TB. We report a high-resolution structure of the acyltransferase (AT) domain of Pks13 at $2.59 \AA$ A resolution. Structural comparison with the full-length AT domain (PDB code, 3TZW and 3TZZ) reveals a different orientation of the C-terminal helix and rearrangement of some conserved residues.

\section{Introduction}

Tuberculosis (TB) and its drug-resistant forms are still the primary causes of mortality, surpassing other infectious diseases(Dande \& Samant, 2018) and emphasizing the unmet clinical need for new drugs with novel mechanisms. Owing to the indispensable and specific lipids forming the envelope of Mycobacterium tuberculosis (Dubnau et al., 2000), targeting the synthesis and transport pathways of mycolic acids has always been the main route of TB drug discovery(Bhatt et al., 2007; Brennan \& Nikaido, 1995; North et al., 2014; Wilson et al., 2013). 
27 biosynthesis pathway (Gavalda et al., 2009; Portevin et al., 2004), and Pks13 has been extensively

28 studied as a drug target for TB (Aggarwal et al., 2017; Thanna et al., 2016). The type-1 polyketide

29 synthase enzyme Pks13 consists of five domains. The medial three are mandatory polyketide

30 synthase (PKS) domains, namely, the ketoacyl synthase (KS) domain, the acetyltransferase (AT)

31 domain and the acyl carrier protein (ACP) domain. The other ACP domain is adjacent to the KS

32 domain, and the thioesterase (TE) domain is the C-terminal portion of Pks13. The overall Pks13

33 topological structure has the order ACP-KS-AT-ACP-TE (Fig. 1A).

34 The residue $\operatorname{Ser}^{55}$ in the N-ACP domain has been identified as a very important active site for 35 initializing the pathway. The sfp gene encodes Phosphopantetheinyl transferase (PPTase), which modifies ACPs by providing a P-pant arm for the general function of carrying the substrate acyl chain via a thioester bond involving its terminal thiol group(Chalut et al., 2006; Gavalda et al., 2009; Wilson et al., 2013). The meromycoloyl chain on the N-ACP domain is transferred to the

KS domain, and the intermediate product $\alpha$-alkyl $\beta$-ketothioester is produced by a Claisen-type condensation reaction with another substrate, the carboxyacyl-CoA loaded by the AT domain. The mycolic acid precursor generated by the $\mathrm{C}$-terminal $\mathrm{ACP}$ domain is then released by the TE domain (Abrahams \& Besra, 2016; Dubey et al., 2002).

43 Despite increasing insights into the mechanism of Pks13, no full-length structural information

44 has been reported, except that the structures of a few domains belonging to Pks 13 have been solved (Bergeret et al., 2012; Herbst et al., 2016).

Here, we report a high-resolution structure of the core motif of the AT domain. First, the fulllength Pks13 protein was successfully purified, and an extended crystal screening was performed, in which the initial crystal was obtained. While attempting to phase the diffraction data of the crystal, we found that the crystalized protein suggested a degraded fragment. Then, the crystals 
50 were solved, and the N-terminal sequence was identified by mass spectrometry, the results of

51 which were in line with the phase presented by the Se-Met crystal dataset. These results indicated

52 that the crystalized protein was actually proteolyzed to become a fragment ( $\mathrm{Leu}^{717}$ to $\left.\operatorname{Arg}^{826}\right)$. The

53 overall crystal structure displayed a fold similar to the reported AT domain, excluding several

54 conformational changes relative to the reported AT domain (Protein Data Bank codes: 3TZW,

55 3TZZ). The structural alignment performed by the secondary structure matching (SSM) in Coot

56 also showed a superimposition of the core motif and the AT domain with an r.m.s.d. of $1.33 \AA$,

57 which was mainly attributed to the rearrangement of residues $\mathrm{Ala}^{796}-\mathrm{Ser}^{801}$. In addition, the

58 position of residue $\mathrm{Ser}^{801}$ that is reported to be the catalytic residue was shifted away from the

59 active site (Bergeret et al., 2012; Gavalda et al., 2009). Furthermore, a highly conserved arginine

60 residue, $\operatorname{Arg}^{826}$, lost a hydrogen bond with the side chain of $\mathrm{Gln}^{773}$, as observed in our structure.

61 These features might all contribute to the unique state that survived proteolysis.

62 We believe that comprehensive structural studies of Pks13 will pave the way for structure-based

63 antimycobacterial drug design and drug screening.

64 Materials \& Methods

65 Cloning, Over-expression, and Purification

66 The codon-optimized gene encoding the full-length Pks13 protein originating from

67 Mycobacterium tuberculosis was ligated into the $N d e 1$ and Xho 1 sites of the pET-28b expression

68 plasmid (Novagen, Madison, WI, USA). The sfp gene, which encodes the P-pant transferase that

69 serves as a kind of cofactor to modify $\mathrm{Ser}^{55}$ in the N-ACP domain of Pks13, from Bacillus subtilis

70 str.168(Chalut et al., 2006) was also ligated into the Nde 1 and Xho 1 sites of the pET-21b

71 expression plasmid (Novagen, Madison), and a terminator codon was added to the C-terminal end. 
72 The detailed information on these constructs is shown in Table 1. All constructed plasmids were

73 verified by sequencing.

74 The constructed plasmid pks13-pET-28b was cotransformed with sfp-pET-21b into E. coli 75 strain BL21 (DE3). The bacteria containing these recombinant plasmids were grown at $310 \mathrm{~K}$ in 76 M9 medium (6 g/L Na $\mathrm{HPO}_{4}, 3 \mathrm{~g} / \mathrm{L} \mathrm{KH}_{2} \mathrm{PO}_{4}, 1 \mathrm{~g} / \mathrm{L} \mathrm{NH} \mathrm{N}_{4} \mathrm{Cl}, 0.5 \mathrm{~g} / \mathrm{L} \mathrm{NaCl}$, and $0.4 \%$ glucose) 77 supplemented with $0.05 \mathrm{~g} / \mathrm{L}$ kanamycin and $0.1 \mathrm{~g} / \mathrm{L}$ ampicillin. When the OD600 reached 0.5 , the 78 medium was supplemented with amino acids $(0.1 \mathrm{~g} / \mathrm{L}$ L-lysine, L-phenylalanine, and L-threonine; $790.05 \mathrm{~g} / \mathrm{L}$ L-isoleucine, L-leucine, and L-valine; and $0.1 \mathrm{~g} / \mathrm{L} \mathrm{L}$-Se-methionine). In addition, the 80 protein was overexpressed after the addition of $0.3 \mathrm{mM}$ IPTG at $289 \mathrm{~K}$ for approximately $16 \mathrm{~h}$.

81 Cell pellets were harvested by 4,000 rpm centrifugation for $10 \mathrm{~min}$ and suspended in a solution of $821 \mathrm{mM}$ PMSF, $150 \mathrm{mM} \mathrm{NaCl}$, and $25 \mathrm{mM}$ Tris/ $\mathrm{HCl}(\mathrm{pH} 8.0)$ suspension buffer. After sonication, 83 we clarified the cell lysate by centrifugation at $15,000 \mathrm{~g}$ for $30 \mathrm{~min}$. The supernatant containing 84 the modified protein was applied to a nickel-affinity column (Ni-NTA; GE Healthcare) 85 preequilibrated with suspension buffer.

86 The resin was gradient washed with ice-cold washing buffer ( $25 \mathrm{mM} \mathrm{Tris} / \mathrm{HCl}(\mathrm{pH} 8.0)$ and 150 $87 \mathrm{mM} \mathrm{NaCl}$ ) containing 20,30 , and $40 \mathrm{mM}$ imidazole, and the proteins were eluted with elution 88 buffer (25 mM Tris/ $\mathrm{HCl} \mathrm{pH} \mathrm{8.0,150} \mathrm{mM} \mathrm{NaCl,} \mathrm{and} 250 \mathrm{mM}$ imidazole). Before loading onto an 89 anion exchange column (Source Q; GE Healthcare), the eluate with $250 \mathrm{mM}$ imidazole was diluted 90 by half with buffer A ( $25 \mathrm{mM}$ Tris/ $\mathrm{HCl}(\mathrm{pH} 8.0)$ and $3 \mathrm{mM} \mathrm{DTT})$. Subsequently, the peak fractions 91 were collected for further purification by size-exclusion chromatography (Superdex 200 10/300; 92 GE Healthcare) in $10 \mathrm{mM}$ Tris/ $\mathrm{HCl}(\mathrm{pH} 8.0)$ buffer containing $100 \mathrm{mM} \mathrm{NaCl}$. The purity of the 93 protein was determined by $12 \%$ SDS-PAGE gels stained by Coomassie brilliant blue. The eluted 
94 protein was concentrated by a $10 \mathrm{kDa}$ centrifugal filter and flash-frozen in liquid nitrogen for

95 crystallization.

\section{Crystallization}

97 The protein encoded by the constructed plasmid and labeled with Se-Met was concentrated to $12 \mathrm{mg} / \mathrm{ml}$. Index (Hampton Research) and PEG/ION (Hampton Research) kits were used for the

99 initial crystallization trials at $293 \mathrm{~K}$ by the sitting-drop vapor-diffusion method(Luft \& Detitta,

100 1995). Each drop contained $1 \mu \mathrm{L}$ of protein solution and an equal volume of reservoir solution.

101 The initial crystal was obtained from a solution of $300 \mathrm{mM} \mathrm{KAc,} \mathrm{pH} 8.1$ and 20\% PEG 3,350.

102 Further crystal optimization experiments were performed by systematic variation of the precipitant 103 concentration. Ultimately, the best crystals were screened in a solution consisting of $300 \mathrm{mM} \mathrm{KAc}$,

$104 \mathrm{pH} \mathrm{8.1}$, and 25\% PEG 3,350. The crystals grew to full size in 10 days and were flash-frozen in 105 liquid nitrogen with $10 \%$ glycerol added as a cryoprotectant before X-ray diffraction.

\section{Data collection}

107 X-ray diffraction data were collected at $100 \mathrm{~K}$ using a Pilatus3 6M detector. All the datasets

108 were obtained at beamline BL19U1 of the Synchrotron Radiation Facility in Shanghai(Wang et 109 al., 2016). A total of 360 images were recorded with $0.5 \mathrm{~s}$ exposure at a crystal-to-detector distance 110 of $450 \mathrm{~mm}$, and a total rotation range of $360^{\circ}$ was covered using 1.0 oscillation.

\section{Protein $\mathrm{N}$-termial sequence based on mass spectrometry}

112 Regarding the dataset of the crystalized pks13, the initial trial did not seem to provide a structure

113 with all of the residues because of the insufficient density for many residues. After X-ray

114 diffraction, the crystals were collected together and analyzed with SDS-PAGE gels stained by

115 Coomassie brilliant blue. The gel with a single low molecular line was processed with the standard 
116 in-gel digestion for mass spectrometric characterization to identify the actual location of the

117 degraded fragment in Pks13(Shevchenko et al., 2006).

\section{Data refinement}

119 All datasets were processed by HKL-2000(Brodersen et al., 2006). The crystal structure of the

120 motif was solved by single-wavelength anomalous dispersion (SAD) phasing using the anomalous

121 data collected from the Se-Met crystal. The final model was manually built in Coot(Emsley et al.,

122 2010) and refined in PHENIX(Adams et al., 2010). The final models were validated by MolProbity

123 and deposited in the Protein Data Bank (PDB code 5XUO).

\section{Results}

\section{Purification and crystallization of Pks13}

126 The full-length Pks13 protein was successfully overexpressed in E. coli BL21 (DE3), and the 127 initial crystal condition (300 mM KAc, pH 8.1 and 20\% PEG 3,350) was screened. The mature 128 lump-like crystals were optimized after a series of crystal optimization experiments, including crystallization with different detergents and additives.

\section{Data collection}

131 X-ray diffraction datasets for the Se-Met-labeled crystals were obtained at beamline BL19U1 132 of the Synchrotron Radiation Facility in Shanghai with a wavelength of $0.97852 \AA$. Diffraction 133 images for the crystals were processed using HKL-2000.

\section{Protein N-terminal sequence}

135 The prepared gel was digested by trypsin, and the digestion was purified into freeze-dried 136 peptide powder. Then, the peptide was resolved by an Orbitrap Elite LC-MS/MS for analysis. The

137 sequenced peptides were blasted within the full-length pks13 protein, and the crystalized fragment 138 protein was located in the range from $\mathrm{Ala}^{717}$ to $\operatorname{Arg}^{826}$ (Table 2). 
139

140

141

142

143

144

146

147

148

149

150

151

152

153

154

156

157

\section{Data refinement}

The crystal belonged to the space group R32, with asymmetric unit cell parameters of $\mathrm{a}=93.694$, $b=93.694, c=97.908, \alpha=\beta=90$, and $\gamma=120$. Additionally, the phases were determined by the SAD method. The final model was manually built in Coot and refined in PHENIX to an $R_{\text {free }}$ of $26.05 \%$ with good stereochemistry. The collected and processed data are presented in Table 3.

\section{Overall architecture and Superimposition with AT domain}

The overall structure of the core motif contains a long $\alpha$ helix, five short $\alpha$ helixes and two short $\eta$ turns, in the order of $\alpha 1-\alpha 2-\alpha 3-\alpha 4-\eta 1-\eta 2-\alpha 5-\alpha 6$, which constitutes a compact motif (Fig. 1B). The long $\alpha$ helix, $\alpha 4$, distributes in the middle and is surrounded by the other five short $\alpha$ helixes and two short $\eta$ turns (Fig. 2A). Superimposition with the reported structure of the AT domain (PDB code 3TZZ)(Bergeret et al., 2012) suggested that the core motif was located in the central region of the AT domain (Fig. 2B). The crystallized core motif ranging from $\mathrm{Leu}^{717}$ to $\mathrm{Arg}^{826}$ represents approximately one-third of the AT domain, and the overall crystal structure displays a fold similar to the reported AT domain (Fig. 2C).

Although sequence alignment showed 100\% identity between the core motif and the AT domain, the secondary structure elements presented a slight conformational change from residues $\mathrm{Ala}^{796}$ to $\mathrm{Ser}^{801}$, for which refinement indicated two $\eta$ turns instead of the $\beta$ strand highlighted by red dashed square line (Fig. 3A). According to the structure of the AT domain reported by Bergeret et al., there was a parallel six-stranded $\beta$-sheet $(\beta 13-\beta 12-\beta 4-\beta 5-\beta 10-\beta 11)$ along with the active site in the reported AT domain, while only the central $\beta$ strand, $\beta 5$, was presented in the motif structure and was refined as a completely different secondary element (Fig. 3B, 3C). Previous studies suggested that the conserved $\operatorname{Ser}^{801}$ and $\operatorname{Arg}^{826}$ could serve as a catalytic residue and binding site, respectively. The active site $\operatorname{Ser}^{801}$ of the AT domain is located in the nucleophilic elbow between 
$162 \beta 5$ and helix $\alpha 10$ and could directly contact the lipid substrate. Additionally, the active cite

163 constituted the part of the highly conserved consensus sequence Gly-X-Ser-X-Gly that stabilizes

164 the $\beta 5$ strand shape(Bergeret et al., 2012; Serre et al., 1995). In our work, the topographic

165 conformation of $\mathrm{Ala}^{796}$ to $\mathrm{Ser}^{801}$ was transformed into two relatively disordered $\eta$ turns, along with

166 the conformational change of the position of $\mathrm{Ser}^{801}$ dislocating from the substrate. Furthermore,

167 the side chain of the binding site $\operatorname{Arg}^{826}$ was also stretched to the reverse side of $\alpha 5$ and lost its

168 interaction with Gln ${ }^{773}$. However, this side chain formed direct hydrogen bonds with the negative

169 side chain of the lipid substrate, and the conformation was held in position through a strong

170 hydrogen bond interaction with the side chain of $\mathrm{Gln}^{773}$ in the AT domain (Fig. 4A).

171 The structural alignment performed by SSM in Coot(Emsley \& Cowtan, 2004) also showed that

172 the superimposition of the core motif and the AT domain had an r.m.s.d. of $1.33 \AA$; along with the

173 conformational changes, this alignment might also suggest a more compact crystal packing state

174 than that of the AT domain. According to a close view of the superimposition, some particular

175 apolar contacts among $\alpha 1$ ( $\left.\mathrm{His}^{723}, \mathrm{Leu}^{730}\right)$ and the long $\alpha 4\left(\mathrm{Gln}^{773}, \mathrm{Ile}^{779}, \mathrm{Gln}^{780}\right.$, and $\left.\mathrm{Leu}^{783}\right)$ and

$176 \alpha 5\left(\mathrm{Ile}^{823}\right)$ residues all contribute to the stabilization of the unique state (Fig. 4B). Electrostatic

177 calculations of the AT domain (Protein Data Bank code 3TZZ) revealed the presence of an

178 electropositive area corresponding to the floor of the active site cavity due to the presence of Ser ${ }^{801}$

179 and $\operatorname{Arg}^{826}$ (Fig. 4C). Comparison of the electrostatic potential surface presentation of the motif

180 indicated that the surface of the active site cavity was transformed to an electronegative state (Fig.

$1814 \mathrm{D})$

\section{Discussion}

183 The synthesis and transport pathways of mycolic acids in Mycobacterium tuberculosis have

184 always been a critical drug target. These mycolic acids serve as the primary defense to counteract 
185 the low permeability of the envelop to many hydrophilic molecules. Many biochemical and

186 structural studies have sought to elucidate the participation of Pks13 in the synthesis of the lipid

187 complex. Obtaining the structure of Pks13 is of great significance in drug screening, as many

188 inhibitors have been reported to target $\mathrm{Pks} 13$ or its individual domains.

189 The structure of the fragment of the AT domain provides a relatively new perspective of a unique

190 state that can evade proteolysis. We have determined the $2.59 \AA$ high-resolution crystal structure

191 of a partial AT domain from the Mycobacterium tuberculosis Pks13 protein. The overall structure

192 of the core motif of the AT domain is similar to the corresponding part of the reported AT domain,

193 with slight conformational differences. Some conserved residues showed a completely different

194 secondary structure. Residues $\mathrm{Ala}^{796}, \mathrm{Val}^{797}, \mathrm{Ile}^{798}, \mathrm{Gly}^{799}, \mathrm{Gln}^{800}$, and $\mathrm{Ser}^{801}$ formed a $\beta$ strand in

195 the previously reported AT domain (PDB codes 3TZW and 3TZZ), which instead refined as a

196 flexible loop conformation in the motif structure. In contrast to the typical structure of the whole

197 AT domain containing a palm-shaped parallel six-stranded $\beta$ sheet, in which $\beta 5$ is located in the

198 middle of a connection with the other five $\beta$ strands. In our work, the $\beta$-sheet structure was

199 disrupted along with loosing connections among these $\beta$ strands due to the conformational

200 changes. Actually, there was less possibility of the AT domain remaining the same because of the

201 conformational changes from $\mathrm{Ala}^{796}$ to $\mathrm{Ser}^{801}$, which tend to confirm the speculation that the

202 conformational changes are a tactic to evade proteolysis. With the structural alignment performed

203 by SSM in Coot, the superimposition of the core motif and the AT domain shows an r.m.s.d. of

$2041.33 \AA$. The novel packed structure formed by these bundles seems tighter than the AT domain,

205 which is especially reflected in the apolar contacts among $\alpha 1$ (His ${ }^{723}$, Leu ${ }^{730}$ ) and the long $\alpha 4$

$206\left(\mathrm{Gln}^{773}, \mathrm{Ile}^{779}, \mathrm{Gln}^{780}\right.$, and $\left.\mathrm{Leu}^{783}\right)$ and $\alpha 5\left(\mathrm{Ile}^{823}\right)$ residues. These apolar contacts among the 
207 residues might strengthen the interactions of $\alpha 4$ with other helixes to form a more stable packing 208 state.

209 Additionally, the active site $\mathrm{Ser}^{801}$, which plays a critical role in catalytic activity, was dislocated

210 away from the substrate cavity to the inner position of the core motif. The nucleophilic elbow of

$211 \alpha 10$ and $\beta 5$ also transformed from an electropositive state to an electronegative state which

212 indicates an unsuitable state to absorb a substrate. In summary, the conformational change of

213 residues from $\mathrm{Ala}^{796}$ to $\mathrm{Ser}^{801}$ and the rearrangement of residues $\mathrm{Gln}^{773}, \mathrm{Ser}^{801}$ and $\mathrm{Arg}^{826}$ might all

214 suggest that the degraded fragment formed a unique crystal packing state to survive proteolysis.

215 In other words, the fragment forms a relatively stable state in contrast to the AT domain in such

216 conditions. This work might provide new insight into the core motif of the AT domain. Our work

217 also provides a structural basis for protein engineering.

218 However, the overall structure of Pks13 is still unrevealed, and its mechanism is unknown yet.

219 More work should be performed, and we hope that our present work will provide some assistance.

220 Acknowledgments: We gratefully acknowledge the assistance of the staff of BL19U1 at the 221 Shanghai Synchrotron Radiation Facility (SSRF) for their assistance with X-ray diffraction data 222 collection.

223 References

224 Abrahams KA, and Besra GS. 2016. Mycobacterial cell wall biosynthesis: a multifaceted 225

226

227

228

229

230

231

232

233 antibiotic target. Parasitology:1-18.

Adams PD, Afonine PV, Bunkóczi G, Chen VB, Davis IW, Echols N, Headd JJ, Hung LW, Kapral GJ, and Grossekunstleve RW. 2010. PHENIX: a comprehensive Python-based system for macromolecular structure solution. Acta Crystallogr D Biol Crystallogr 66:213221.

Aggarwal A, Parai MK, Shetty N, Wallis D, Woolhiser L, Hastings C, Dutta NK, Galaviz S, Dhakal RC, and Shrestha R. 2017. Development of a Novel Lead that Targets M. tuberculosis Polyketide Synthase 13. Cell 170:249-259.

Bergeret F, Gavalda S, Chalut C, Malaga W, Quémard A, Pedelacq JD, Daffé M, Guilhot C, Mourey L, and Bon C. 2012. Biochemical and structural study of the atypical acyltransferase 
235

236

237

238

239

240

241

242

243

244

245

246

247

248

249

250

251

252

253

254

255

256

257

258

259

260

261

262

263

264

265

266

267

268

269

270

271

272

273

274

domain from the mycobacterial polyketide synthase Pks13. Journal of Biological Chemistry 287:33675-33690.

Bhatt A, Molle V, Besra GS, Jr JW, and Kremer L. 2007. The Mycobacterium tuberculosis FAS-II condensing enzymes: their role in mycolic acid biosynthesis, acid-fastness, pathogenesis and in future drug development. Molecular Microbiology 64:1442-1454.

Brennan PJ, and Nikaido H. 1995. The Envelope of Mycobacteria. Annual Review of Biochemistry 64:29-63.

Brodersen DE, La Fortelle ED, Vonrhein C, Bricogne G, Nyborg J, and Kjeldgaard M. 2006. Applications of single-wavelength anomalous dispersion at high and atomic resolution. Acta Crystallographica 56:431-441.

Chalut C, Botella L, De S-DAC, Houssin C, and Guilhot C. 2006. The nonredundant roles of two 4'-phosphopantetheinyl transferases in vital processes of Mycobacteria. Proc Natl Acad Sci U S A 103:8511-8516.

Dande P, and Samant P. 2018. Acquaintance to Artificial Neural Networks and use of artificial intelligence as a diagnostic tool for tuberculosis: A review. Tuberculosis 108:1.

Dubey VS, Sirakova TD, and Kolattukudy PE. 2002. Disruption of msl3 abolishes the synthesis of mycolipanoic and mycolipenic acids required for polyacyltrehalose synthesis in Mycobacterium tuberculosis $\mathrm{H} 37 \mathrm{Rv}$ and causes cell aggregation. Molecular Microbiology 45:1451-1459.

Dubnau E, Chan J, Raynaud C, Mohan VP, Lanéelle MA, Yu K, Quémard A, Smith I, and Daffé M. 2000. Oxygenated mycolic acids are necessary for virulence of Mycobacterium tuberculosis in mice. Molecular Microbiology 36:630-637.

Emsley P, and Cowtan K. 2004. Coot: model-building tools for molecular graphics. Acta Crystallographica 60:2126-2132.

Emsley P, Lohkamp B, Scott WG, and Cowtan K. 2010. Features and development of Coot. Acta Crystallographica 66:486-501.

Gavalda S, Léger M, Rest BVD, Stella A, Bardou F, Montrozier H, Chalut C, Burletschiltz O, Marrakchi H, and Daffé M. 2009. The Pks13/FadD32 Crosstalk for the Biosynthesis of Mycolic Acids in Mycobacterium tuberculosis. Journal of Biological Chemistry 284:1925519264.

Herbst DA, Jakob RP, Zähringer F, and Maier T. 2016. Mycocerosic acid synthase exemplifies the architecture of reducing polyketide synthases. Nature 531:533.

Luft JR, and Detitta GT. 1995. Chaperone salts, polyethylene glycol and rates of equilibration in vapor-diffusion crystallization. Acta Crystallographica 51:780-785.

North EJ, Jackson M, and Lee RE. 2014. New Approaches to Target the Mycolic Acid Biosynthesis Pathway for the Development of Tuberculosis Therapeutics. Current Pharmaceutical Design 20:-.

Portevin D, De SAC, Houssin C, Grimaldi C, Chami M, Daffé M, and Guilhot C. 2004. A polyketide synthase catalyzes the last condensation step of mycolic acid biosynthesis in mycobacteria and related organisms. Proc Natl Acad Sci U S A 101:314-319. 
Serre L, Verbree EC, Dauter Z, Stuitje AR, and Derewenda ZS. 1995. The Escherichia coli malonyl-CoA:acyl carrier protein transacylase at 1.5-A resolution. Crystal structure of a fatty acid synthase component. Journal of Biological Chemistry 270:12961-12964.

Shevchenko A, Tomas H, Havli J, Olsen JV, and Mann M. 2006. In-gel digestion for mass spectrometric characterization of proteins and proteomes. Nature Protocols 1:2856-2860.

Thanna S, Knudson SE, Grzegorzewicz A, Kapil S, Goins CM, Ronning DR, Jackson M, Slayden RA, and Sucheck SJ. 2016. Synthesis and evaluation of new 2-aminothiophenes against Mycobacterium tuberculosis. Organic \& Biomolecular Chemistry 14:6119.

Wang Z, Pan Q, Yang L, Zhou H, Xu C, Yu F, Wang Q, Huang S, and He J. 2016. Automatic crystal centring procedure at the SSRF macromolecular crystallography beamline. Journal of Synchrotron Radiation 23:1323-1332.

Wilson R, Kumar P, Parashar V, Vilchèze C, Veyronchurlet R, Freundlich JS, Barnes SW, Walker JR, Szymonifka MJ, and Marchiano E. 2013. Antituberculosis thiophenes define a requirement for Pks13 in mycolic acid biosynthesis. Nature Chemical Biology 9:499. 


\section{Figure 1}

The overall Pks13 domain structure has the order ACP-KS-AT-ACP-TE.

A. The leading domain N-ACP (25-91) is colored orange. The medial three domains are mandatory PKS domains, including the KS domain (118-539), the AT domain (715-1018) and the C-ACP domain (1241-1304), colored cyan, green and orange, respectively. The TE domain (1470-1727) is located in the $C$ terminus and colored blue. Residue numbers are given below for each domain boundary. B. The motif resides in the AT domain ranging from Ala717 to Arg826. The whole topographic structure is composed of $6 \alpha$ helixes and 2 short $\eta$ turns, in the order of $N$ terminus- $\alpha 1-\alpha 2-\alpha 3-\alpha 4-\eta 1-\eta 2-\alpha 5-\alpha 6-C$ terminus.

A

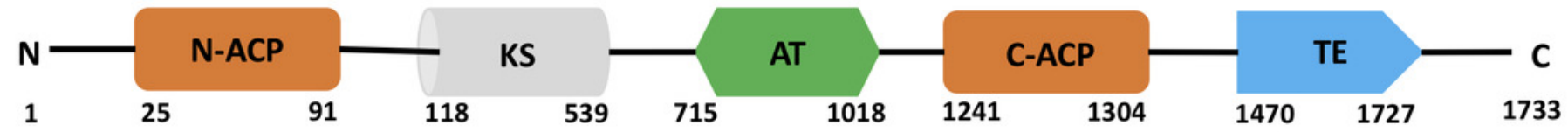

B

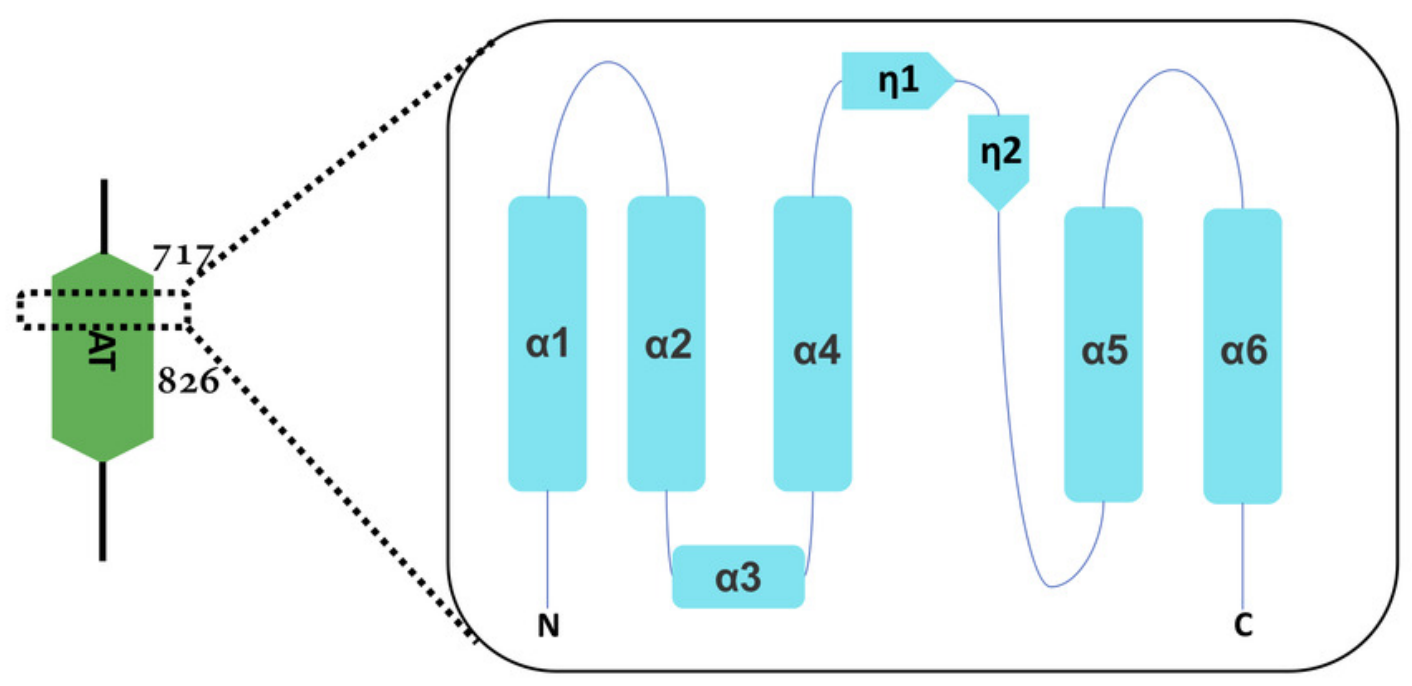




\section{Figure 2}

The architecture of the core motif from the AT domain and structural comparison with the AT domain (PDB code 3TZW)

A. The overall topological structure of the core motif contains a long $\alpha$ helix in the middle, five short $\alpha$ helixes and two short $\eta$ turns around the long helix, which constitutes a compact motif. The top and bottom views with a rotation of $90^{\circ}$ are exhibited on the right. $B$. The structures of the motif and the AT domain (PDB code 3TZZ) are superimposed together and colored blue and gray, respectively. The aligned region is zoomed in for clear observation. C. The superimposition of the motif and the region of the AT domain that could be aligned is shown in two orthogonal views. The secondary elements are labeled in the picture.

A
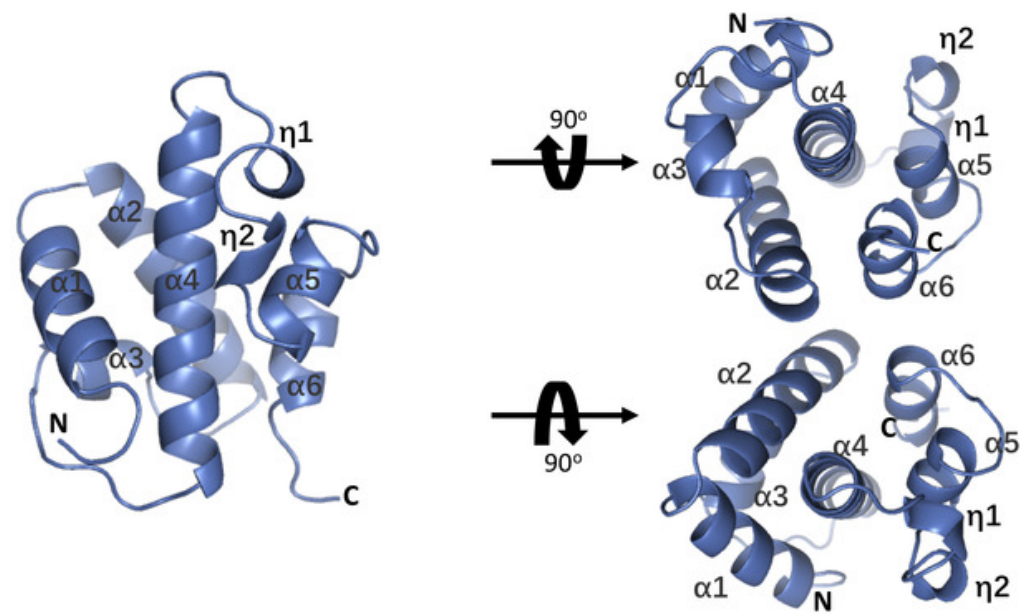

B

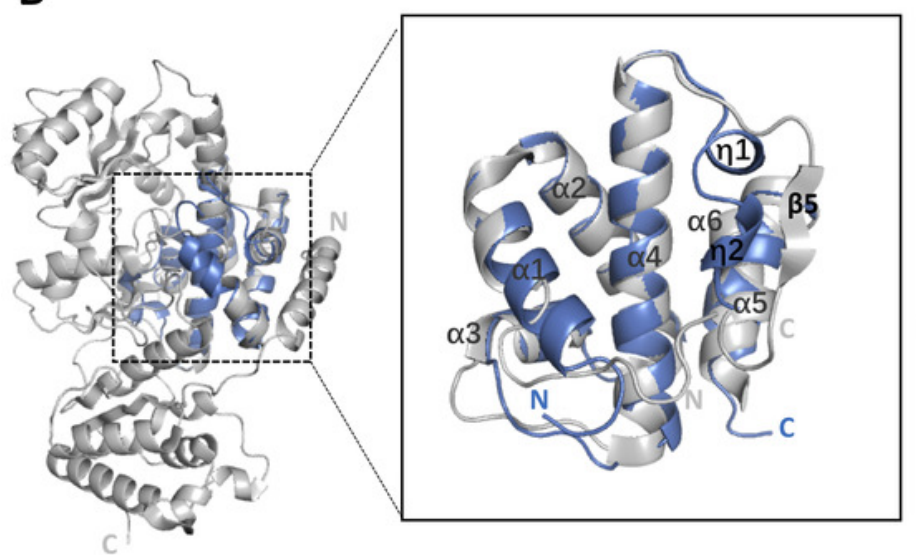

Peer] reviewing PDF | (2017:10:21429:2:0:NEW 7 Apr 2018)
C

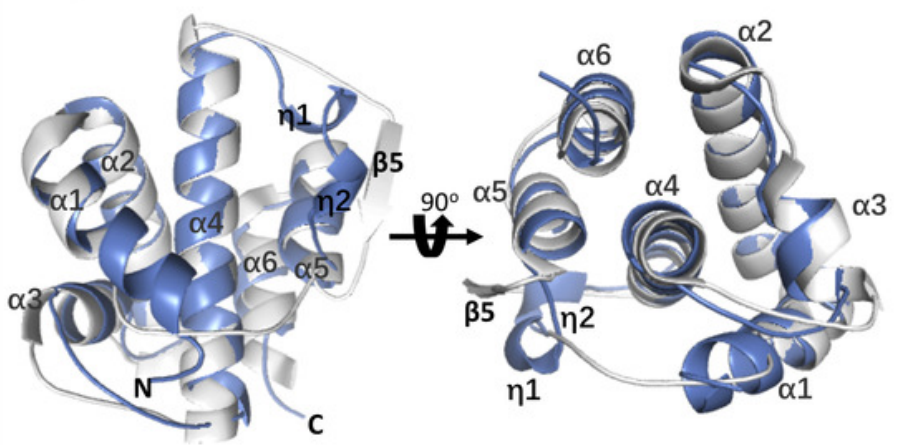




\section{Figure 3}

Structural and sequence alignments.

A. Structure-based sequence alignment with the whole AT domain. The secondary structural elements of the motif are given along the top of the alignment; the secondary elements of the AT domain (Protein Data Bank codes 3TZZ and 3TZW) are shown below. The difference between the structures is circled by the red dashed line. $B$. The six $\beta$-sheets ( $\beta 13-\beta 12-\beta 4-\beta 5$ $\beta 10-\beta 11)$ (Protein Data Bank codes 3TZW and 3TZZ) are presented in the figure, and $\beta 5$ is aligned with the $\eta$ turns of the motif. C. Some conserved residues show a totally different topographic structure, and residues Lys793, Pro794, Ala795, Ala796, Val797, Ile798, Gly799, Gln800, and Ser801 are shown as sticks. 
A

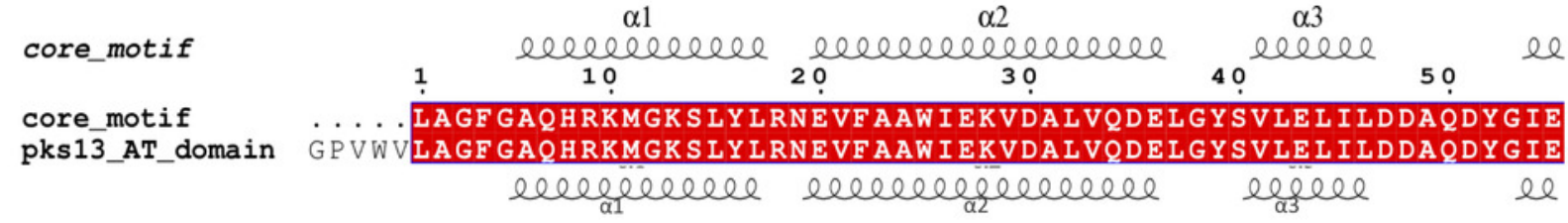

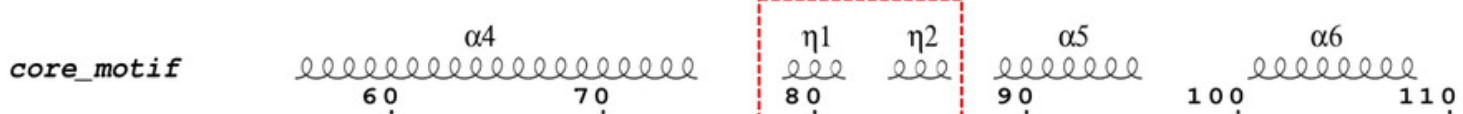

core_motif TTQVTIFAIQIALGELLRHHGAKP AAVIGQSLGEAASAYFAGGLSLRDATRAICS. . . .

pks13_AT_domain TTQVTIFAIQIALGELLRHHGAKPAAVIGQS J_GEAASAYFAGGLS LRDATRAICSRSH H M elebelelebelel

\section{elebel}

a5

B

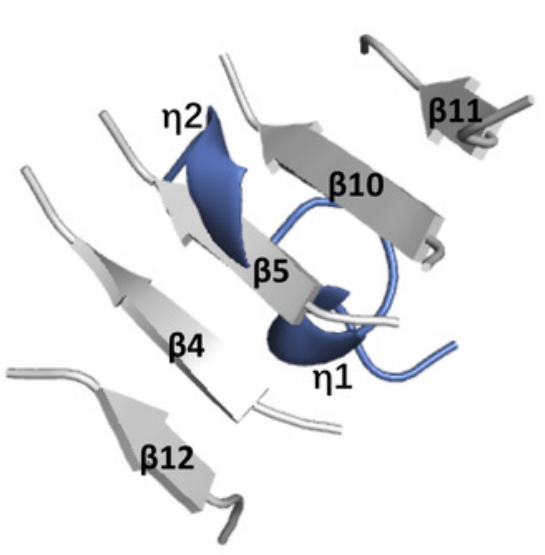




\section{Figure 4}

Structural comparison between different states of AT domain.

A. Detailed description of the active site of the motif compared with the AT domain. The nucleophilic elbow comprising strand $\beta 5$ and helix $\alpha 10$ in the AT domain corresponding to helix $\alpha 5$ and two helical $\eta$ turns, respectively, is shown in cartoon representation. Important residues defining the active site are shown and labeled. Hydrogen bonds are represented by orange dotted lines. The lipid substrate colored orange is shown as sticks. B. Some apolar contacts among $\alpha 1$ (His723, Leu730) and the long $\alpha 4$ (Gln773, Ile779, Gln780, Leu783) and a5 (Ile823) residues are shown and labeled. C. Electrostatics calculations for the AT domain (Protein Data Bank code 3TZZ) revealed the presence of an electropositive area corresponding to the floor of the active site cavity bound with a lipid substrate. The surface representation was generated by PyMOL and colored according to its electrostatic potential (positive potential, blue; negative potential, red). The substrate cavity was highlighted by a dotted red circle and zoomed at the right panel. D. Electrostatics calculations for the motif in this work revealed the electrostatic potential transformation from an electropositive state to an electronegative state. The substrate originating from the AT domain (Protein Data Bank code 3TZZ) was docked on the floor of the catalytic cavity and was highlighted with a red dotted circle. 

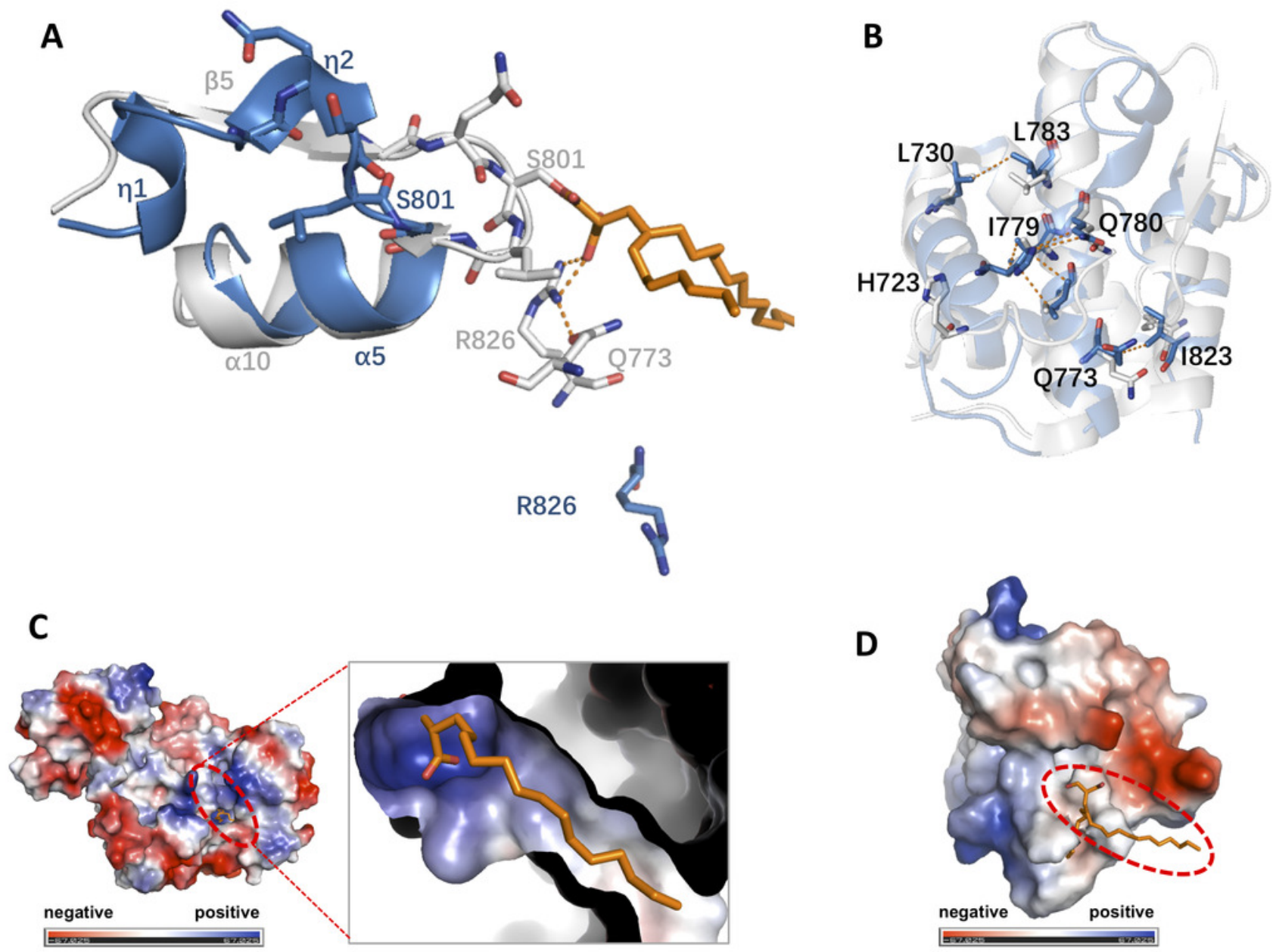
Table $\mathbf{1}$ (on next page)

Macromolecule production information 
Table 1 Macromolecule production information

\begin{tabular}{ccc}
\hline Source organism & Mycobacterium & Bacillus subtilis str.168 \\
tuberculosis(H37Rv) & \\
DNA source & Full-length Pks13 & Sfp (P-pant transferase) \\
Forward primer & 5-ggattccatatgatggcagatgtggccg-3 & 5-ggaattccatatgaagatttacggaa - \\
Reverse primer & 5-ccgctcgagctgtttaccaacctcg-3 & 5-ccgctcgagtcaagcggaagcgata-3 \\
Cloning vector & pET-28b & pET-21b \\
Expression vector & pET-28b & pET-21b \\
Expression host & E. coli strain(DE3) & E. coli strain(DE3) \\
Complete amino & MADVAESQENAPAERA......IEADRTSEV & MKIYGIYMDRPLSOEENERF \\
acid sequence of & GKQLE & MSFISPEKREKCR.....PGYK \\
the construct & & MAVCAAHPDFPEDITMVSY \\
produced & & EELL \\
\hline
\end{tabular}

3

4 


\section{Table 2 (on next page)}

Mass spectrum based on protein $\mathrm{N}$-terminal sequencing

PEP, Posterior Error Probability of the identification. This value essentially operates as a pvalue, where smaller is better. 
Table 2 Mass spectrum based on protein $\mathrm{N}$-terminal sequencing

\begin{tabular}{|c|c|c|c|c|c|}
\hline Sequence & Length & Mass & Charges & PEP & Score \\
\hline AGFGAQHR & 8 & 842.9 & $2 ; 3$ & 0 & 201.07 \\
\hline AGFGAQHRK & 9 & 971.07 & 3 & 0.020363 & 8.308 \\
\hline \multicolumn{6}{|l|}{ HHGAKPAAVIGQSLGEA } \\
\hline & 29 & 2835.478 & $2 ; 3 ; 4 ; 5$ & $2.88 \mathrm{E}-48$ & 83.418 \\
\hline \multicolumn{6}{|l|}{ ASAYFAGGLSLR } \\
\hline \multicolumn{6}{|l|}{ HHGAKPAAVIGQSLGEA } \\
\hline & 33 & 3278.6909 & $3 ; 4 ; 5$ & 0.012023 & 14.817 \\
\hline \multicolumn{6}{|l|}{ ASAYFAGGLSLRDATR } \\
\hline KMGKSLYLR & 9 & 1094.627 & $2 ; 3$ & 0.0011449 & 41.427 \\
\hline MGKSLYLR & 8 & 966.53207 & 2 & $5.16 \mathrm{E}-09$ & 75.109 \\
\hline \multicolumn{6}{|l|}{ MGKSLYLRNEVFAAWIE } \\
\hline & 18 & 2154.1296 & $3 ; 4$ & $1.24 \mathrm{E}-11$ & 40.856 \\
\hline \multicolumn{6}{|l|}{ K } \\
\hline NEVFAAWIEK & 10 & 1205.6081 & 2 & $8.84 \mathrm{E}-19$ & 89.08 \\
\hline \multicolumn{6}{|l|}{ PAAVIGQSLGEAASAYFA } \\
\hline & 24 & 2305.2066 & $2 ; 3$ & $1.27 \mathrm{E}-303$ & 269.26 \\
\hline \multicolumn{6}{|l|}{ GGLSLR } \\
\hline \multicolumn{6}{|l|}{ PAAVIGQSLGEAASAYFA } \\
\hline & 28 & 2748.4195 & 3 & $6.29 \mathrm{E}-159$ & 160 \\
\hline \multicolumn{6}{|l|}{ GGLSLRDATR } \\
\hline SLYLRNEVFAAWIEK & 15 & 1837.9727 & $2 ; 3$ & $7.27 \mathrm{E}-68$ & 127.02 \\
\hline SSGLVPR & 7 & 714.40244 & 2 & 3.61E-08 & 74.191 \\
\hline
\end{tabular}

2 


\section{Table 3 (on next page)}

X-ray data collection and refinement statistics 
Table 3. X-ray data collection and refinement statistics.

\begin{tabular}{|c|c|}
\hline Data Set & Core motif of AT domain \\
\hline \multicolumn{2}{|l|}{ Data collection } \\
\hline X-ray source & SSRF BEAMLINE BL19U1 \\
\hline Space group & R32 \\
\hline Wavelength $(\AA)$ & 0.97852 \\
\hline Resolution Range $(\AA ̊)$ & $50-2.587$ \\
\hline Total No. of reflections & 90229 \\
\hline No. of unique reflections & $5358(524)$ \\
\hline Completeness (\%) & 99.70 \\
\hline Redundancy & 16.999 \\
\hline R r.i.m. & 0.026 \\
\hline$I / \sigma(I)$ & $41.27(2.10)$ \\
\hline \multicolumn{2}{|l|}{ Refinement } \\
\hline Resolution range & $50-2.59$ \\
\hline Reflections: working/test & $5083 / 276$ \\
\hline Final $R_{\text {cryst }}$ & $23.69 \%$ \\
\hline
\end{tabular}




\begin{tabular}{lc}
\hline Final $R_{\text {free }}$ & $26.05 \%$ \\
rotamer outliers & $2.4 \%$ \\
Ramachandran plot: & \\
favored/allowed/outliers (\%) & $93.58 / 4.59 / 1.83$ \\
Rmsd bonds $(\AA)$ & 0.003 \\
Rmsd angles $\left({ }^{\circ}\right)$ & 0.540 \\
PDB accession code & $5 \times U O$ \\
\hline
\end{tabular}

4

5

6 